\title{
ARBITRAJE Y CONTRATACIÓN PÚBLICA EN SERVICIOS DE SALUD
}

\author{
FRANK GARCÍA AsCENCIOS \\ Universidad de Lima, Lima, Perú \\ Recibido: 17/2/2020 - Aprobado: 24/2/2020 \\ doi: https://doi.org/10.26439/iusetpraxis2020.n50-51.5053
}

\begin{abstract}
RESUMEN. El arbitraje en la contratación pública ha tenido un colosal desarrollo en el país, debido a que generalmente los conflictos que involucran contratos con el Estado se resuelven mediante arbitraje. En el presente trabajo se aborda un tema escasamente conocido y desarrollado: el arbitraje en materia de contratación pública en servicios de salud. Este tipo de arbitraje se presenta en las controversias derivadas de los contratos entre el Estado y los establecimientos de salud para brindar atención médica a los asegurados. El Estado celebra contratos como lafas pública (EsSalud o SIS) con Ipress (clínicas). Este es un arbitraje especializado en salud, en el cual se aplica esencialmente la Ley de Contrataciones del Estado, su reglamento y la normativa sanitaria correspondiente. El trabajo brinda ideas centrales con base en la experiencia del autor.
\end{abstract}

PALABRAS CLAVE: arbitraje / contratación pública / salud / lafas e Ipress / asegurados / Superintendencia Nacional de Salud / Centro de Conciliación y Arbitraje

\section{ARBITRATION AND PUBLIC PROCUREMENT IN HEALTH SERVICES}

ABSTRACT. Arbitration in public procurement has had a colossal huge development in the country, because generally conflicts involving contracts with the State are generally resolved through arbitration. This paper addresses a poorly known and developed topic: arbitration in public procurement in health service public procurements. This type of arbitration occurs in disputes arising from contracts between the State and health facilities, to provide medical care to for the insured. The State enters into contracts as a public lafas (EsSalud or SIS) with Ipress (clinics). This is an arbitration specialized in health, where the State Contracting Public Procurement Law, its rRegulations and the corresponding health regulations are essentially applied. This research e work provides central ideas based on the author's experience.

KEYWORDS: arbitration / public contracting procurement / health / lafas and Ipress / insured / National Superintendency of Health / Conciliation and Arbitration Center 


\section{INTRODUCCIÓN}

El arbitraje como mecanismo alternativo de solución de conflictos goza de un creciente apogeo en el país. Sarcásticamente, se afirma que hoy en día todos quieren ser árbitros debido a que no hay nada mejor que decidir. Todos buscan tener la última palabra.

El creciente enamoramiento ha sido impulsado por el arbitraje en contratación pública. Disciplina en la que hay mucho pan por rebanar, a causa de las numerosas posiciones y constantes modificaciones normativas. Sin embargo, no hay nada escrito en materia de arbitraje y contratación pública en servicios de salud.

Arbitraje, en que se aplica la normativa en salud, la Ley de Contratación Pública y su reglamento. Una materia especializada que ha generado la participación de otro órgano ajeno al Organismo Supervisor de las Contrataciones del Estado (OSCE). Ahora, el Centro de Conciliación y Arbitraje (Ceconar) de la Superintendencia Nacional de Salud (Susalud) participa gestionándolo.

Las siguientes líneas buscan brindar un primer acercamiento a este tipo de arbitraje especializado.

\section{ARBITRAJE EN CONTRATACIÓN PÚBLICA}

El arbitraje es un mecanismo heterocompositivo de resolución de conflictos en el que un tercero - llamado árbitro- conoce y resuelve controversias de libre disposición sometidas a su competencia. Es un mecanismo alternativo al Poder Judicial en cuanto a solución de conflictos. La Constitución le otorga rango constitucional y lo regula como jurisdicción especial'. Más allá de discutir su naturaleza jurisdiccional, el tema central es que su reconocimiento constitucional como jurisdicción ha permitido su fortalecimiento y consolidación en el sistema jurídico nacional.

En el Perú, el arbitraje ha tenido un desarrollo titánico debido a la crisis del sistema de justicia estatal, que ha ocasionado que se adopten otros sistemas alternativos para solucionar los conflictos, desde la difusión de la conciliación extrajudicial, la mediación y el trato directo hasta el arbitraje.

Si bien hay características o ventajas que distinguen al arbitraje del clásico proceso judicial - como son la especialidad, la flexibilidad, la celeridad, entre otras-, consideramos que su central fortaleza radica en la libertad para someterse a este mecanismo alternativo. Autonomía que permite evaluar si es conveniente sustraerse del poder estatal de justicia y elegirlo como medio idóneo para resolver conflictos.

1 "Artículo 139 de la Constitución.- Son principios y derechos de la función jurisdiccional:

1. La unidad y exclusividad de la función jurisdiccional. No existe ni puede establecerse jurisdicción alguna independiente, con excepción de la militar y la arbitral". 
La libertad para someterse al arbitraje se materializa en la autonomía para decidir si celebrar o no un convenio arbitral, el cual representa la confianza en el arbitraje; la luz verde para adoptar este mecanismo alternativo. El convenio arbitral

provoca un doble efecto: uno positivo y otro negativo. Genera un efecto positivo, pues las controversias serán resueltas en un proceso arbitral, donde las partes deberán cumplir con lo laudado. Asimismo, produce un efecto negativo, ya que otros órganos están impedidos de solucionar estas controversias. (García, 2013, p. 84)

La libertad para decidir si acudir al arbitraje es su real fortaleza. Compartimos las palabras de Emmanuel Gaillard (2010) cuando afirma que "las nociones, esencialmente filosóficas, de libertad y de voluntad se encuentran en el corazón mismo del derecho de arbitraje" (p. 8).

La noción de libertad para someterse al arbitraje tiene distinto matiz cuando se aborda el arbitraje en la contratación pública, debido a que en el Perú, desde 1998 con la derogada Ley 26850 (Ley de Contrataciones y Adquisiciones del Estado), el legislador adopta su obligatoriedad como mecanismo para resolver las controversias en materia de contratación estatal.

La obligatoriedad del uso del arbitraje para resolver los conflictos en la ejecución contractual o la interpretación del contrato público sugiere pensar que el legislador se ha alejado de la noción de libertad. Sin embargo, no es el caso, ya que el Estado ha adoptado la decisión de que, frente a cualquier conflicto con algún contratista, entonces - como regla general- debe resolverse mediante arbitraje.

La libertad permite que uno decida si acudirá o no al arbitraje. Esta misma libertad, sumada a la problemática del sistema estatal de justicia, hizo que el Estado opte por el camino de someter a arbitraje sus conflictos derivados de la ejecución del contrato.

Sobre el particular, García-Calderón (2001) sostiene que

es evidente que cualquiera que desee contratar con el Estado se verá afectado y deberá someterse a esta obligatoriedad en la medida en que se desee contratar por ser una exigencia interna de éste, tal como puede ocurrir con cualquier empresa privada que exige requisitos mínimos para poder contratar con terceros. (p. 144)

En ese sentido, la central fortaleza del arbitraje basado en la libertad continúa vigente en nuestro sistema jurídico, tanto en el arbitraje privado como en el de contratación pública.

La Constitución reconoce la libertad para acudir al arbitraje en contratación pública. El artículo 62 señala que "los conflictos derivados de la relación contractual solo se solucionan en la vía arbitral o judicial, según los mecanismos de protección previstos en el contrato o contemplados en la ley". Seguidamente, el artículo 63 acoge lo siguiente: 
El Estado y las demás personas de derecho público pueden someter las controversias derivadas de relación contractual a tribunales constituidos en virtud de tratados en vigor. Pueden también someterlas a arbitraje nacional o internacional, en la forma en que lo disponga la ley.

El artículo 4 del Decreto Legislativo 1071 (Decreto Legislativo que norma el Arbitraje) regula la competencia del Estado para someter a arbitraje las controversias derivadas de sus contratos. Asimismo, la Ley de Contrataciones del Estado establece que "las controversias que surjan entre las partes sobre la ejecución, interpretación, resolución, inexistencia, ineficacia o invalidez del contrato se resuelven mediante conciliación o arbitraje, según el acuerdo de las partes".

Respecto a la experiencia del arbitraje en la contratación pública, consideramos que es positiva, a pesar de que en los últimos años se ha cuestionado la conducta no ética de algunos árbitros, lo cual ha llevado a fortalecer el control sobre los propios árbitros, las instituciones arbitrales, los procuradores públicos y todo agente vinculado al sistema.

Finalmente, el arbitraje y la contratación pública son dos áreas disímiles, pero que en el Perú se han hermanado. En efecto, los especialistas en ambas materias deben conocer la normativa vigente del arbitraje en general y de la Ley de Contrataciones del Estado y su reglamento. Los árbitros están atentos a los cambios -o propuestas de reformas- en la referida ley.

\section{CONTRATACIÓN PÚBLICA EN SERVICIOS DE SALUD}

El contrato público es definido como el acuerdo celebrado entre el Estado y un privado para la adquisición de bienes y servicios, y la ejecución de obras. Tafur y Miranda (2008) lo conceptualizan en los siguientes términos:

Según este tipo de contratos el Estado (a través de sus diversas entidades) puede satisfacer sus necesidades de provisión de bienes, servicios y obras, que le permitan cumplir con sus objetivos. Bajo este tipo de contratos se engloban los de compraventa, suministro, arrendamiento, contratación de servicios, consultorías, ejecución de obras, y otros de similar naturaleza. Los contratos regidos por estas normas tienen un denominador común: el Estado entrega dinero y recibe a cambio un bien, servicio u obra. (p. 147)

Generalmente, la experiencia arbitral en contratación pública radica en los conflictos que se presentan sobre bienes, servicios, ejecución y consultoría de obras. Sin embargo, existe un área escasamente difundida que se ha presentado en los últimos años: las controversias derivadas de los servicios contratados entre el Estado y los establecimientos médicos privados con el objeto de brindar servicios de salud a los asegurados.

El contrato celebrado entre el Estado y los establecimientos médicos privados permite que los asegurados del Seguro Social de Salud (EsSalud) y el Seguro Integral 
de Salud (SIS) puedan atenderse en clínicas privadas, por lo que, como Estado, se busca descongestionar los hospitales y otorgar un mejor servicio de salud a los pacientes. Dicha política está acorde con el objetivo de hacer efectivo el derecho a la salud.

La salud es un derecho inherente al ser humano. Uno, por la condición de persona, tiene garantizado este derecho, que permite que se logre vivir en bienestar físico y mental. El concepto de salud trasciende al solo estado de enfermedad. La Organización Mundial de la Salud (OMS) la define como un "bienestar físico, mental y social, y no solamente la ausencia de afecciones o enfermedades".

El derecho a la salud es uno que goza de reconocimiento expreso en la Constitución. El artículo 9 indica que "el Estado determina la política nacional de salud. El Poder Ejecutivo norma y supervisa su aplicación. Es responsable de diseñarla y conducirla en forma plural y descentralizadora para facilitar a todos el acceso equitativo a los servicios de salud". Mientras que el artículo 10 regula que "el Estado reconoce el derecho universal y progresivo de toda persona a la seguridad social, para su protección frente a las contingencias que precise la ley y para la elevación de su calidad de vida". Finalmente, el artículo 11 legisla lo siguiente:

El Estado garantiza el libre acceso a prestaciones de salud y a pensiones, a través de entidades públicas, privadas o mixtas. Supervisa asimismo su eficaz funcionamiento. La ley establece la entidad del Gobierno nacional que administra los regímenes de pensiones a cargo del Estado.

En ese contexto, la Ley 26842 (Ley General de Salud) regula la responsabilidad del Estado en garantizar la adecuada cobertura de salud de la población. El artículo VI del Título Preliminar establece que

es de interés público la provisión de servicios de salud, cualquiera sea la persona o institución que los provea. Es responsabilidad del Estado promover las condiciones que garanticen una adecuada cobertura de prestaciones de salud a la población, en términos socialmente aceptables de seguridad, oportunidad y calidad.

Es responsabilidad del Estado garantizar la cobertura de salud de la población. Por consiguiente, el aparato público, a través de los mecanismos contractuales vigentes, debe brindar el acceso a la salud mediante su propia infraestructura y a través del sector privado.

En tal sentido, la Ley 29344 (Ley Marco de Aseguramiento Universal en Salud) es fundamental en este objetivo, debido a que, aparte de buscar concretar el acceso universal a la salud, también regula a los agentes vinculados al sector, como el Ministerio de Salud (Minsa), las Instituciones Administradoras de Fondos de Aseguramiento en Salud (lafas) y las Instituciones Prestadoras de Servicio de Salud (Ipress). 
Así, el Decreto Supremo 008-2010-SA (reglamento de la Ley Marco de Aseguramiento Universal en Salud) norma el sistema de contratación entre lafas e Ipress, que tiene por objetivo garantizar el acceso a los servicios de salud. El artículo 19 indica que

las IAFAS tendrán libertad para contratar con las IPRESS de su elección para la conformación de sus redes de prestación de servicios de salud. Las IAFAS deberán contratar la prestación de servicios de salud única y exclusivamente con instituciones prestadoras de servicios de salud registradas en SUNASA (SUSALUD), de acuerdo a las normas que ésta establezca. (2010)

Las lafas son entidades públicas, privadas o mixtas que gestionan fondos para atenciones en salud. En cambio, las Ipress son establecimientos de salud y servicios médicos públicos, privados o mixtos que brindan una atención médica (prevención, promoción, diagnóstico, tratamiento y rehabilitación). En otras palabras, Ipress es todo centro médico².

La interrelación entre lafas e Ipress es fundamental para finalmente brindar atención en salud a los asegurados. Esta relación de naturaleza contractual tiene sus complejidades por las normas médicas aplicables, como las normas técnicas aprobadas por el Minsa, entre otras que las partes deciden que sean vinculantes para la ejecución del contrato.

La complejidad de este tipo de contratación es mayor cuando la lafas es pública y la Ipress es privada. EsSalud y SIS son lafas públicas que, dentro del marco de esta normativa, celebran contratos con Ipress privadas para ofrecer atención médica a sus asegurados.

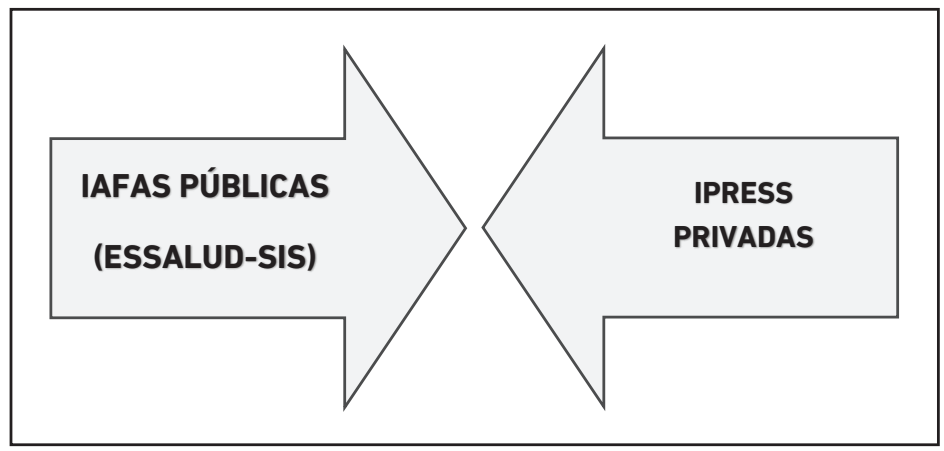

Figura 1. lafas públicas -Ipress privadas

Elaboración propia

2 El Decreto Legislativo 1158, norma destinada al fortalecimiento y cambio de denominación de la Superintendencia Nacional de Aseguramiento en Salud, regula en mayor detalle a las lafas e Ipress. 
Este es un contrato especializado al que, adicionalmente, se aplica la Ley de Contrataciones del Estado y su reglamento, por lo que existen en el país arbitrajes sobre prestaciones de servicios de salud contratadas por lafas públicas e Ipress privadas. Toda una novedad, debido a que ahora se aplica la normativa de contratación pública en los contratos de prestación de servicios médicos a asegurados.

El entorno del contrato entre lafas públicas e Ipress privadas se enmarca en uno de contratación pública. El contrato de servicios de salud deriva de un procedimiento especial de contratación. Diversos decretos supremos ${ }^{3}$ regulan disposiciones destinadas a viabilizar la contratación entre EsSalud y SIS e Ipress a través de un procedimiento especial. Sin embargo - a la vez-, no debe dejarse de lado su especialidad en el sector salud, que lo convierte en un tipo de arbitraje especializado en salud.

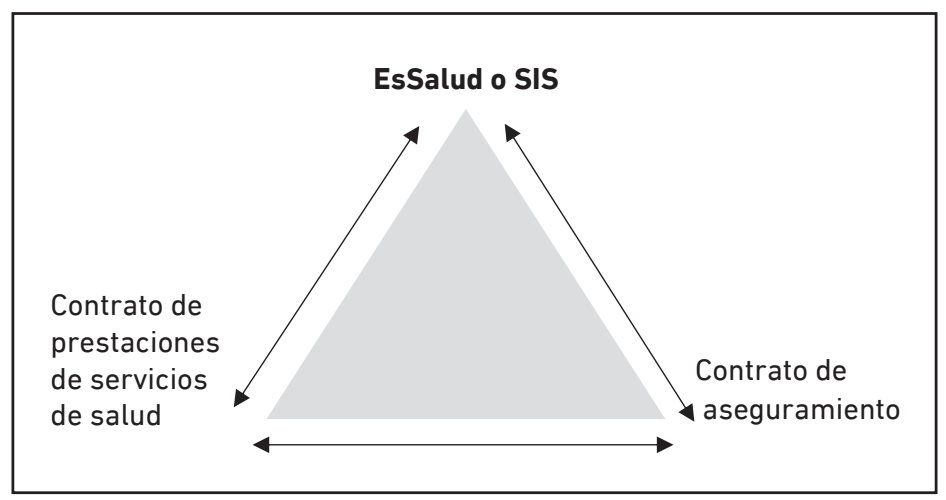

Figura 2. Contrato de prestaciones de servicios de salud lafas públicas e Ipress privadas Elaboración propia

\section{EXPERIENCIA ARBITRAL}

La experiencia arbitral en esta materia aún es escasa, pero no inexistente. El Ceconar de Susalud fue el centro arbitral competente para administrar estos casos, conforme a la normativa emitida para viabilizar este procedimiento especial de contratación. Voluntad del sector que ha sido plasmada en los contratos de servicios de salud entre EsSalud o el SIS con los diversos establecimientos privados de salud.

El Ceconar es un órgano dependiente administrativamente de Susalud que gestiona con autonomía los mecanismos alternativos de resolución de conflictos en salud (MARCS)

3 Entre los decretos supremos que regulan este procedimiento especial de contratación, tenemos los correspondientes a los números 002-2013-SA, 013-2014-SA, 017-2014-SA, entre otros. 
a través de la mediación, la conciliación extrajudicial, el arbitraje y demás mecanismos alternativos. Asimismo, administra a nivel nacional el servicio de conciliación y arbitraje especializado en salud (García, 2018, p. 24).

La decisión de someter este tipo de casos a un arbitraje institucional administrado solo por Ceconar genera controversias, debido a que se deja de lado a los centros arbitrales privados. Sobre todo cuando es esencialmente en contratación pública. Materia extraña para Ceconar, centro acostumbrado a gestionar arbitrajes en atenciones médicas, seguros de salud, Seguro Obligatorio de Accidentes de Tránsito (SOAT) y Seguro Complementario de Trabajo de Riesgo (SCTR).

El Ceconar ha gestionado estos arbitrajes con la carencia de no ser un centro especializado en contratación pública. Asimismo, genera un caso sui generis en el país, puesto que es el segundo centro público - diferente a OSCE- que gestiona arbitrajes en contratación pública. Incluso con mayor inflexibilidad que OSCE, porque implementa el arbitraje institucional público y exclusivo para gestionar estos casos.

De igual modo, la escasa experiencia del Ceconar en este arbitraje que involucra un contrato público ha generado la nula transparencia para publicar los laudos arbitrales emitidos en esta materia, pese a que una institución arbitral pública debe transparentar dicha información, al ser una de las partes el Estado y al hallarse en controversia un caso sobre contratación estatal.

Dejando a un lado las dificultades en la gestión de estos arbitrajes, debe precisarse que este es un arbitraje de derecho, en razón de que se está frente a una controversia en contratación pública.

Esta experiencia restringida al arbitraje institucional del Ceconar ha sido modificada en los últimos contratos entre lafas públicas e Ipress privadas, en que se han pactado progresivamente convenios arbitrales con la propuesta de administración de instituciones arbitrales privadas.

Las controversias que se dilucidan en este arbitraje son frecuentemente las siguientes: a) aplicación de penalidades, b) falta de pago, c) incumplimiento contractual, d) indemnización por daños, e) resolución contractual, f) recepción y conformidad, entre otras.

La controversia respecto a las penalidades se presenta continuamente debido a que los contratos entre lafas públicas e Ipress privadas regulan la aplicación de "otras penalidades" dentro del marco de la Ley de Contrataciones del Estado, es decir, el contrato recoge un número de conductas tipificadas como sancionables, la frecuencia de la conducta, la forma de cálculo y el procedimiento a aplicar para sancionar.

Ahora, en estos tiempos de pandemia global debido al COVID-19, se ha generado otra controversia en estos contratos de prestación de servicios de salud, ya que no se 
han podido brindar los servicios con la normalidad prevista. Es necesario resaltar que, generalmente, la forma de pago en estos contratos es la tarifa per cápita, es decir, el pago se realiza por la atención a un número promedio de asegurados de la lafas pública estimado en el contrato, y no por cada prestación individualizada. En contraste, respecto a los contratos en que sí se recoge un pago por un número determinado de prestaciones, no existe mayor contingencia.

En relación con la condición de árbitro único o el presidente del tribunal arbitral, debe tener la especialidad en arbitraje, contratación pública y derecho administrativo, conforme a la Ley de Contrataciones del Estado. En ese sentido, al designar el Ceconar al árbitro - que generalmente es árbitro único-, debe tener en consideración este mandato de carácter legal respecto a los profesionales que conocen y resuelven controversias en materia de contratación estatal.

En lo que respecta a las actuaciones arbitrales, el arbitraje administrado por Ceconar se inicia con la demanda arbitral. Sin embargo, previendo estos casos en contratación pública, en la que se regula el plazo de caducidad de treinta días hábiles para iniciarlo, el reglamento arbitral del Ceconar ${ }^{4}$ establece la posibilidad de presentar la solicitud arbitral, pero en los quince días siguientes de ingresada dicha solicitud tiene que alcanzarse la demanda.

La demanda arbitral se traslada por un plazo de quince días a la contraparte. En caso de que el demandado deduzca alguna excepción o reconvención, se traslada al demandante para su absolución. Una vez absuelta, en caso de que las partes no lleguen a un acuerdo sobre quién es el árbitro, siempre que sea resuelta por un árbitro único, entonces Ceconar lo designa. En caso de que la cláusula regule la conformación de un tribunal arbitral, entonces los dos árbitros designan al presidente del tribunal.

Previo abono de honorarios arbitrales, se convoca a una audiencia única, donde el árbitro se instala e invita a las partes a llegar a un acuerdo antes de continuar con el arbitraje. Si no se alcanza, entonces el árbitro difiere o se pronuncia sobre las excepciones deducidas, de ser el caso. Luego, se fijan los puntos controvertidos, conforme a la demanda y la contestación. Seguidamente, se admiten, rechazan u ordenan de oficio pruebas. En caso de que no hubiera pruebas que actuar, en el acta se concluye con la etapa probatoria y se concede un plazo de cinco días a las partes para que presenten sus

4 “Resolución de Superintendencia N.`162-2016-SUSALUD/S

Sexto. Solicitud Arbitral

Excepcionalmente, cuando se regule plazos de caducidad y/o prescripción menor o igual a quince (15) días para iniciar una acción legal, entonces se podrá iniciar el arbitraje con la sola presentación de la solicitud arbitral, debiendo presentar la demanda arbitral en un plazo no mayor quince (15) días de ingresada la solicitud, bajo apercibimiento de archivar el expediente arbitral.

Esta excepción debe ser probada en la solicitud arbitral, salvo contrario se archiva por parte de la secretaría del Centro". 
alegatos escritos. En caso de que sí existieran, entonces aquellas se tienen que actuar. Finalmente, se pone el caso para laudar.

Respecto a los arbitrajes administrados por instituciones arbitrales privadas, no es necesario presentar la demanda arbitral, sino la solicitud arbitral, que se pondrá en conocimiento de la contraparte.

En relación con el laudo arbitral, el árbitro tiene un plazo regulado en el reglamento arbitral para emitirlo. Este debe notificarse por el Sistema Electrónico de Contrataciones del Estado (Seace) y a ambas partes, conforme lo regula la Ley de Contratación Pública. No obstante, al no estar registradas las bases de contratación en el Seace por ser, generalmente, un procedimiento especial de contratación, entonces no puede efectivizarse la notificación por dicho medio, lo que genera que el árbitro tenga que ordenar que la secretaría arbitral remita copia del laudo al OSCE.

En las siguientes figuras se puede observar la tramitación del arbitraje en el Ceconar:

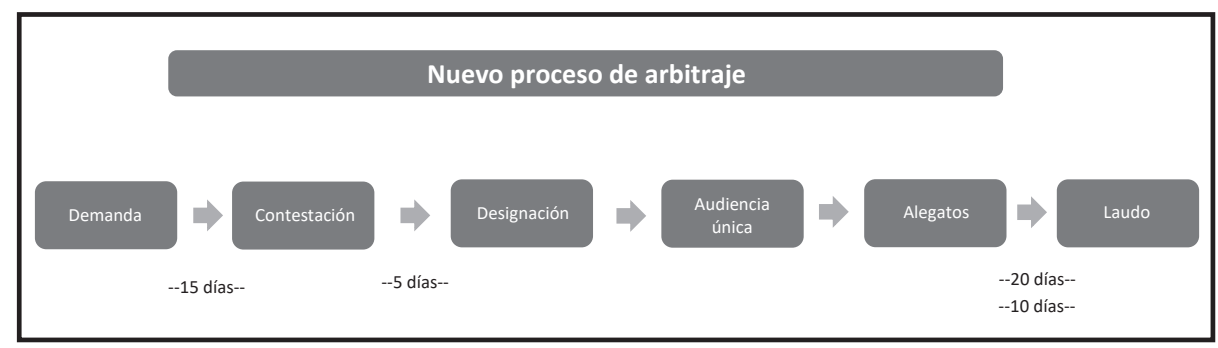

Figura 3. Tramitación

Elaboración propia

\section{Nuevo proceso de arbitraje con excepciones y reconvención}

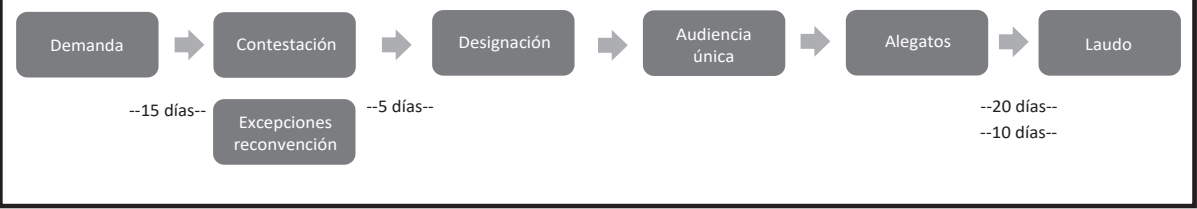

Figura 4. Tramitación con excepciones y reconvención

Elaboración propia 
Más allá de las controversias que puedan suscitarse entre lafas públicas e Ipress privadas, resulta imprescindible tener en consideración que debe fortalecerse este vínculo contractual en beneficio de los asegurados. La alternativa de contar con clínicas privadas para que atiendan a los asegurados y descongestionen los hospitales públicos es positiva, sobre todo en un sistema sanitario con limitaciones como el peruano (Soto, 2019).

\section{CONCLUSIONES}

Se espera que estas líneas hayan servido como un primer acercamiento a este arbitraje especializado, en el cual el objeto del contrato no es la provisión de bienes ni servicios en general, ni tampoco de obras, sino la debida prestación de servicios de salud a través de un centro médico privado a favor de los asegurados de EsSalud y SIS.

Lo más cercano al típico arbitraje en contratación pública es la participación del Estado como parte y la aplicación de la Ley de Contrataciones y su reglamento. No obstante, su especialidad radica en el objeto del contrato, que lleva a que también se tengan en consideración las normas técnicas médicas y demás normativa sanitaria aplicable en cada contrato en particular.

La normativa vigente ha colocado primero al Ceconar como única institución arbitral pública que gestiona estos arbitrajes. Ello fue cuestionable al no considerar otros centros arbitrales privados para administrar estos casos. Sin embargo, progresivamente, se han ido celebrando convenios arbitrales en los que encargan su administración a instituciones arbitrales privadas.

El presente texto ha buscado brindar algunos elementos centrales sobre las nociones y la práctica arbitral en los conflictos de servicios de salud. Con el deseo de haber aportado algunas nociones fundamentales sobre este arbitraje especializado, se concluyen estas líneas.

\section{REFERENCIAS}

Constitución Política del Perú. (1993). Recuperado de https://lpderecho.pe/constitucion -politica-peru-actualizada/

Decreto Supremo 008-2010-SA, Decreto Supremo que aprueba el reglamento de la Ley 29344, Ley Marco de Aseguramiento Universal en Salud. (2 de abril del 2010). Recuperado de http://www.sunat.gob.pe/legislacion/remunerac/DecSup008.pdf

Gaillard, E. (2010). Teoría jurídica del arbitraje internacional. Asunción: La Ley ParaguayaCentro de Estudios de Derecho, Economía y Política-Thomson Reuters.

García, F. (2013). El convenio arbitral en el derecho peruano. Athina, (10), 83-94. Recuperado de https://revistas.ulima.edu.pe/index.php/Athina/article/viewFile/1160/1114 
García, F. (2018). Mecanismos alternativos de solución de conflictos en seguros de salud en el Perú (tesis de maestría). Universidad Pontificia de Salamanca, España.

García-Calderón, G. (2001). Análisis del arbitraje en la Ley de Contrataciones y Adquisiciones del Estado. lus et Praxis, (032), 142-149. https://doi.org/10.26439/ iusetpraxis2001.n032.3640

Ley 29344, Ley Marco de Aseguramiento Universal en Salud. (9 de abril del 2009). Recuperado de http://www.leyes.congreso.gob.pe/Documentos/Leyes/29344.pdf

Organización Mundial de la Salud. (s. f.). Preguntas más frecuentes. Recuperado de http://www.who.int/suggestions/faq/es/

Resolución de Superintendencia 162-2016-SUSALUD/S. (4 de octubre del 2016). Recuperadodehttps://www.gob.pe/institucion/susalud/normas-legales/851762 -162-2016-susalud-s

Soto, A. (2019). Barreras para una atención eficaz en los hospitales de referencia del Ministerio de Salud del Perú: atendiendo pacientes en el siglo xxI con recursos del siglo xx. Revista Peruana de Medicina Experimental y Salud Pública, 36(2), 304-311. http://dx.doi.org/10.17843/rpmesp.2019.362.4425

Tafur, S. y Miranda, R. (2008). Contratación estatal: algunas reflexiones generales. Derecho \& Sociedad, (29), 140-152. Recuperado de http://revistas.pucp.edu.pe/ index.php/derechoysociedad/article/view/17267 